\section{(2) OPEN ACCESS}

\title{
Comorbidity phenotypes and risk of mortality in patients with ischaemic heart disease in the UK
}

\author{
Francesca Crowe (D) , Dawit T Zemedikun (D) , Kelvin Okoth, Nicola Jaime Adderley (D) , \\ Gavin Rudge, Mark Sheldon, Krishnarajah Nirantharakumar, Tom Marshall
}

- Additional material is published online only. To view please visit the journal online (http://dx.doi.org/10.1136/ heartjnl-2019-316091).

Institute of Applied Health Research, University of Birmingham, Birmingham, UK

\section{Correspondence to} Dr Francesca Crowe, Institute of Applied Health Research, University of Birmingham, Birmingham B15 2TT, UK; F.Crowe@bham.ac.uk

$\mathrm{KN}$ and TM are joint senior authors.

Received 9 October 2019 Revised 27 January 2020 Accepted 28 January 2020 Published Online First 9 April 2020

\section{Linked}

- http://dx.doi.org/10.1136/ heartjnl-2020-316616

\section{Check for updates}

(C) Author(s) (or their employer(s)) 2020. Re-use permitted under CC BY. Published by BMJ.

To cite: Crowe F, Zemedikun DT, Okoth $\mathrm{K}$, et al. Heart 2020;106:810-816.

\section{ABSTRACT}

Objectives The objective of this study is to use latent class analysis of up to 20 comorbidities in patients with a diagnosis of ischaemic heart disease (IHD) to identify clusters of comorbidities and to examine the associations between these clusters and mortality.

Methods Longitudinal analysis of electronic health records in the health improvement network (THIN), a UK primary care database including 92186 men and women aged $\geq 18$ years with IHD and a median of 2 (IQR 1-3) comorbidities.

Results Latent class analysis revealed five clusters with half categorised as a low-burden comorbidity group. After a median follow-up of 3.2 (IQR 1.4-5.8) years, 17645 patients died. Compared with the low-burden comorbidity group, two groups of patients with a highburden of comorbidities had the highest adjusted HR for mortality: those with vascular and musculoskeletal conditions, HR 2.38 ( $95 \% \mathrm{Cl} 2.28$ to 2.49) and those with respiratory and musculoskeletal conditions, HR 2.62 ( $95 \% \mathrm{Cl} 2.45$ to 2.79). Hazards of mortality in two other groups of patients characterised by cardiometabolic and mental health comorbidities were also higher than the low-burden comorbidity group; HR $1.46(95 \% \mathrm{Cl} 1.39$ to 1.52 ) and 1.55 ( $95 \% \mathrm{Cl} 1.46$ to 1.64$)$, respectively. Conclusions This analysis has identified five distinct comorbidity clusters in patients with IHD that were differentially associated with risk of mortality. These analyses should be replicated in other large datasets, and this may help shape the development of future interventions or health services that take into account the impact of these comorbidity clusters.

\section{INTRODUCTION}

Comorbidities, the presence of one or more chronic health condition, among patients with heart disease are becoming increasingly common because of reduced rates of ischaemic heart disease (IHD) case fatality and increased life expectancy. ${ }^{12}$ This places greater pressure on healthcare systems because of the high use of services, ensuing costs and the complexity of managing the healthcare of heart disease patients with many different chronic conditions. ${ }^{3}$ A systematic review has shown that among those with IHD, a greater burden of comorbidities, as measured using the Charlson comorbidity index (CCI), was associated with greater risk of mortality. ${ }^{4}$ This is further supported by studies of patients who had a myocardial infarction (MI) with a smaller number of comorbidities such as cardiometabolic diseases. $^{5}$
While evidence for the impact of a greater burden of comorbidities in patients with IHD on survival is compelling, most studies have used only simple methods to account for the burden of comorbidities. These include the use of a single additive or weighted score such as CCI to quantify the comorbidities, ${ }^{4}$ or combinations of comorbid conditions. ${ }^{56}$ Some of these methods do not distinguish between the same number but different types of comorbidities and will be limited in providing information about underlying pathophysiology that may help optimise interventions in certain groups of patients. ${ }^{78}$ Cluster-based approaches may identify specific patterns of comorbidities associated with higher mortality, but few studies have undertaken such analyses. A recent latent class analysis among MI survivors showed the pattern of comorbidities defined as a high comorbidity cluster with coexisting heart failure, peripheral vascular disease (PVD), hypertension and chronic renal failure had the shortest long-term survival. ${ }^{9}$ This analysis included a large sample size, had a long follow-up and included seven major comorbidities: cerebrovascular disease, chronic obstructive pulmonary disease (COPD) or asthma, diabetes, heart failure, hypertension, renal failure and PVD. However, the effect of other comorbidities such as mental health and musculoskeletal conditions that are common in patients with heart disease ${ }^{1}$ has not been examined before using this data-driven approach. The health improvement network (THIN) database includes general practice (GP) records of 15 million UK patients and offers the opportunity to examine patterns of a number of comorbidities.

The objective of this study is to use latent class analysis of up to 20 comorbidities in patients with a diagnosis of IHD to identify clusters of comorbidities and to examine the associations between these clusters and mortality.

\section{METHODS}

This study was a large open cohort design using THIN primary care database that contains health records for more than 15 million patients from over 750 GP surgeries in the UK. The distribution of age, sex, prevalence of major medical conditions and mortality rates in the THIN cohort is generalisable to the UK population. ${ }^{10}$ Diagnoses in the THIN database are recorded using a hierarchical system called Read codes, which are terms or short phrases used to describe a health-related concept in GP records. ${ }^{11}$ 


\section{Study population}

All data included in this study were from practices that met the acceptable mortality reporting and acceptable computer usage standards-measures of quality assurance for THIN data. ${ }^{12}{ }^{13}$ Patients aged 18 years and older who were registered with a THIN practice contributing data from 1 January 2005 with an incident diagnosis of IHD from 1 January 2005 to 1 January 2015 were included. Person-years of follow-up were calculated from the date of diagnosis of IHD up to whichever came first: exit from the THIN database (transferred practice or died), the last date practice data was collected or 2 December 2015 (study end date).

\section{IHD and comorbidity ascertainment}

Primary care Read codes were used to identify incident diagnoses of IHD, which includes angina, IHD and MI (see online supplementary table 1 ). The presence of comorbidities among patients with IHD was defined as having a diagnosis of any of 20 different comorbidities before the diagnosis of IHD. The 20 comorbidities included other cardiovascular conditions (atrial fibrillation, heart failure, hypertension, PVD or stroke and transient ischaemic attack); respiratory conditions (asthma and COPD); musculoskeletal conditions (osteoarthritis, osteoporosis and rheumatoid arthritis); neurological conditions (dementia and epilepsy); endocrine diseases (diabetes and hypothyroidism); mental health conditions (anxiety, depression and severe mental illness); cancer; kidney disease; and chronic liver disease. The Read codes used for diagnoses of conditions were taken from the Annual Quality and Outcomes Framework (introduced in 2004 for primary care), additional codes identified by searching medical dictionary keywords and online clinical code repositories. ${ }^{14}$ Kidney disease was defined on the basis of estimated glomerular filtration rate (eGFR) $<60 \mathrm{~mL} / \mathrm{min} / 1.73 \mathrm{~m}^{2}$. eGFR was calculated using the Modification of Diet in Renal Disease equation (without the black ethnicity coefficient) from the most recent serum creatinine measurement before the diagnosis of IHD. ${ }^{15}$

\section{Patient and public involvement}

Patients or the public were not involved in setting the research question or the outcome measures, nor were they involved in developing plans for design or implementation of the study. They were not asked to advise on interpretation or writing up of results. There are no plans to disseminate the results of the research.

\section{Statistical analysis}

Latent class analysis was conducted using the 20 comorbidities as observed indicators (see online supplementary text 1 ). Latent class models with two to eight latent classes (clusters) were assessed and a model with five clusters was chosen (see online supplementary table 2). Each individual was assigned to one of the clusters according to their highest computed probability of membership (see online supplementary table 3). Each of the comorbidity clusters was labelled according to the prevalence of comorbidities.

Cox regression models were used to estimate HRs and 95\% CIs of mortality for the five disease clusters using the lowest comorbidity disease cluster as the reference category. Analyses were adjusted for age, sex, quintiles of socioeconomic group (based on Townsend deprivation score), BMI $(<22.5$, 22.5$24.9,25-27.49,27.5-29.9,30-32.49,32.5-34.9$ and $\geq 35 \mathrm{~kg} /$ $\mathrm{m}^{2}$ ) and smoking status (non-smoker, former smoker and current smoker). To account for similarity of outcomes within practices, statistical inference from the Cox regression model was based on cluster-robust standard errors. Smoking status, socioeconomic group and BMI could be missing. To ensure that the same patients were being compared in all analyses, the patients with a missing value for each particular variable were assigned to a separate category for that variable and included in the regression analysis.

In the subgroup analysis, heterogeneity in the associations across the subgroups of patients according to sex, age at IHD diagnosis ( $<65$ vs $\geq 65$ years), smoking status (non-smoker vs former or current smoker), socioeconomic group (quintiles) and BMI ( $<25 \mathrm{vs} \geq 25 \mathrm{~kg} / \mathrm{m}^{2}$ ) was assessed using a standard $\chi^{2}$ test. In sensitivity analysis, patient numbers were restricted to those with complete data on BMI, smoking status and socioeconomic group. An additional analysis was conducted among those with a diagnosis of MI.

All statistical analyses were performed using Stata statistical software V.15. Two-sided p values $<0.05$ were considered statistically significant.

\section{RESULTS}

There were 57088 men and 35098 women with an incident diagnosis of IHD included (table 1). Mean age at diagnosis of IHD was 67 (SD 13) years and the median number of comorbidities was 2 (IQR 1-3). Overall, 84\% had one or more comorbidities and $62 \%$ had two or more comorbidities (see online supplementary figure 2). The distribution of the 20 comorbidities by the five disease clusters are shown in online supplementary table 4. Cluster 1 (51.4\% of patients) was characterised by a low burden of comorbidities; cluster 2 (cardiometabolic; $20.5 \%$ of patients) was dominated by a high proportion of cardiovascular $(98 \%)$ and endocrine conditions (46\%). All patients in cluster 3 (mental health; $11.9 \%$ of patients) had a mental health condition. Cluster 4 (high-burden musculoskeletal cardiovascular; $13.1 \%$ of patients) had high proportions of coexisting cardiovascular (94\%) and musculoskeletal (62\%) conditions. They also had a higher prevalence of dementia (8\%) and cancer (18\%). All patients in cluster 5 (high-burden musculoskeletal respiratory; $3.1 \%$ of patients) had a respiratory condition and over half had a musculoskeletal condition (51\%). They also had a moderate prevalence of mental health conditions (37\%) (figure 1).

Table 1 compares the characteristics of patients with IHD and details of their follow-up for the five comorbidity clusters. Those in the low comorbidity cluster (cluster 1), were younger (63 years), and more likely to be men (72\%), be in the least deprived socioeconomic group, and were the least likely to be prescribed medication for CVD. Individuals with cardiometabolic conditions (cluster 2) had the highest average BMI $\left(29 \mathrm{~kg} / \mathrm{m}^{2}\right)$ and systolic blood pressure $(141 \mathrm{~mm} \mathrm{Hg})$ and were more likely to be prescribed lipid-lowering medication (60\%), antihypertensives (92\%) and aspirin (50\%). Those with mental health conditions (cluster 3) had the highest percent of women (56\%). Individuals in the musculoskeletal cardiovascular cluster (cluster 4) had the highest mean number of comorbidities (4.6), were the oldest (77 years), had the highest proportion of non-smokers $(50 \%)$ and were more likely to be prescribed anticoagulants (19\%) and antiplatelet therapy $(10 \%)$. The musculoskeletal respiratory cluster (cluster 5) had the lowest proportion of non-smokers (13\%), the highest proportion of former (56\%) and current smokers (31\%) and individuals in the most deprived socioeconomic group.

After a median of 3.2 (IQR 1.4-5.8) years of follow-up after a diagnosis of IHD, 17645 patients died (table 2). Survival was 
Table 1 Baseline characteristics of the five comorbidity phenotypes at the time of diagnosis of ischaemic heart disease

\begin{tabular}{|c|c|c|c|c|c|c|c|}
\hline & Low & Cardio-metabolic & Mental health & $\begin{array}{l}\text { High-burden } \\
\text { musculoskeletal } \\
\text { vascular }\end{array}$ & $\begin{array}{l}\text { High-burden } \\
\text { musculoskeletal } \\
\text { respiratory }\end{array}$ & $\mathbf{P}$ & Overall \\
\hline $\mathrm{n}$ & 47413 & 18876 & 10986 & 12049 & 2862 & & 92186 \\
\hline Comorbidities, median (IQR) & 1 (0 to 2$)$ & 3 (2 to 3 ) & 4 (3 to 5$)$ & $4(4$ to 5$)$ & $4(3$ to 5$)$ & & $2(1$ to 3$)$ \\
\hline Comorbidities, mean (SD) & $1.0(0.8)$ & $2.9(0.9)$ & $3.9(1.4)$ & $4.6(1.5)$ & $4.3(1.6)$ & $<0.001$ & $2.3(1.8)$ \\
\hline Age (years) & $62.6(12.0)$ & $70.6(10.9)$ & $63.7(12.2)$ & $77.5(10.0)$ & $71.0(9.9)$ & $<0.001$ & $66.6(12.7)$ \\
\hline BMI $\left(\mathrm{kg} / \mathrm{m}^{2}\right)$, mean (SD) & $27.5(4.9)$ & $29.1(5.6)$ & $28.6(6.0)$ & $27.7(5.7)$ & $26.7(5.8)$ & $<0.001$ & $28.0(5.4)$ \\
\hline $\begin{array}{l}\text { Systolic blood pressure }(\mathrm{mmHg}) \\
\text { mean (SD) }\end{array}$ & $137.4(17.9)$ & $141.0(18.8)$ & $136.7(18.2)$ & $136.3(19.7)$ & $136.3(19.0)$ & $<0.001$ & $137.9(18.5)$ \\
\hline Women, $\mathrm{n}(\%)$ & $13374(28.2 \%)$ & $7793(41.3 \%)$ & $6133(55.8 \%)$ & $6559(54.4 \%)$ & $1239(43.3 \%)$ & $<0.001$ & $35098(38.1 \%)$ \\
\hline Smoking, n (\%) & & & & & & $<0.001$ & \\
\hline Non-smoker & $19384(40.9 \%)$ & $9282(49.2 \%)$ & $4143(37.7 \%)$ & $6059(50.3 \%)$ & $372(13.0 \%)$ & & $39240(42.6 \%)$ \\
\hline Former & $14528(30.6 \%)$ & $7101(37.6 \%)$ & $3482(31.7 \%)$ & $4616(38.3 \%)$ & $1599(55.9 \%)$ & & $31326(34.0 \%)$ \\
\hline Current & $12674(26.7 \%)$ & $2440(12.9 \%)$ & $3322(30.2 \%)$ & $1290(10.7 \%)$ & $887(31.0 \%)$ & & $20613(22.4 \%)$ \\
\hline Missing & $827(1.7 \%)$ & $53(0.3 \%)$ & $39(0.4 \%)$ & $84(0.7 \%)$ & $4(0.1 \%)$ & & $1007(1.1 \%)$ \\
\hline Socioeconomic group, $\mathrm{n}(\%)$ & & & & & & $<0.001$ & \\
\hline Least deprived & $11330(23.9 \%)$ & $4104(21.7 \%)$ & $1982(18.0 \%)$ & $2526(21.0 \%)$ & $397(13.9 \%)$ & & $20339(22.1 \%)$ \\
\hline 2 & $10177(21.5 \%)$ & $3999(21.2 \%)$ & $2001(18.2 \%)$ & $2558(21.2 \%)$ & $461(16.1 \%)$ & & $19196(20.8 \%)$ \\
\hline 3 & $9127(19.3 \%)$ & $3754(19.9 \%)$ & $2173(19.8 \%)$ & $2334(19.4 \%)$ & $589(20.6 \%)$ & & $17977(19.5 \%)$ \\
\hline 4 & $7697(16.2 \%)$ & $3209(17.0 \%)$ & $2203(20.1 \%)$ & $2136(17.7 \%)$ & $641(22.4 \%)$ & & $15886(17.2 \%)$ \\
\hline Most deprived & $5302(11.2 \%)$ & $2145(11.4 \%)$ & $1869(17.0 \%)$ & $1535(12.7 \%)$ & $532(18.6 \%)$ & & $11383(12.4 \%)$ \\
\hline Missing & $3780(8.0 \%)$ & $1665(8.8 \%)$ & $758(6.9 \%)$ & $960(8.0 \%)$ & $242(8.5 \%)$ & & $7405(8.0 \%)$ \\
\hline \multicolumn{8}{|l|}{ Medications } \\
\hline Lipid lowering drugs & $17599(37.1 \%)$ & $11396(60.4 \%)$ & $5217(47.5 \%)$ & $6079(50.5 \%)$ & $1250(43.7 \%)$ & $<0.001$ & $41541(45.1 \%)$ \\
\hline Antihypertensives & $24708(52.1 \%)$ & $17381(92.1 \%)$ & $7730(70.4 \%)$ & $10436(86.6 \%)$ & $2058(71.9 \%)$ & $<0.001$ & $62313(67.6 \%)$ \\
\hline Aspirin & $15701(33.1 \%)$ & $9369(49.6 \%)$ & $4449(40.5 \%)$ & $5410(44.9 \%)$ & $1115(39.0 \%)$ & $<0.001$ & $36044(39.1 \%)$ \\
\hline Oral anticoagulants & $774(1.6 \%)$ & $581(3.1 \%)$ & $295(2.7 \%)$ & $2299(19.1 \%)$ & $183(6.4 \%)$ & $<0.001$ & $4132(4.5 \%)$ \\
\hline Antiplatelet therapies & $3385(7.1 \%)$ & $1619(8.6 \%)$ & $934(8.5 \%)$ & $1157(9.6 \%)$ & $236(8.3 \%)$ & $<0.001$ & $7331(8.0 \%)$ \\
\hline
\end{tabular}

SBP was available for 91219 individuals, and BMI was available for 83230 individuals.

$\mathrm{BMI}$, body mass index.

lowest in clusters 4 and 5, moderate for the cardiometabolic (cluster 2) and mental health (cluster 3) clusters and highest in the low-burden disease cluster (cluster 1) (figure 2). Survival curves for the disease clusters adjusted for age are shown in online supplementary figure 3. After adjusting for age, sex, BMI, smoking and socioeconomic group, the hazard of mortality in all four of the comorbidity clusters was significantly greater than the low comorbidity cluster. The hazard for mortality for

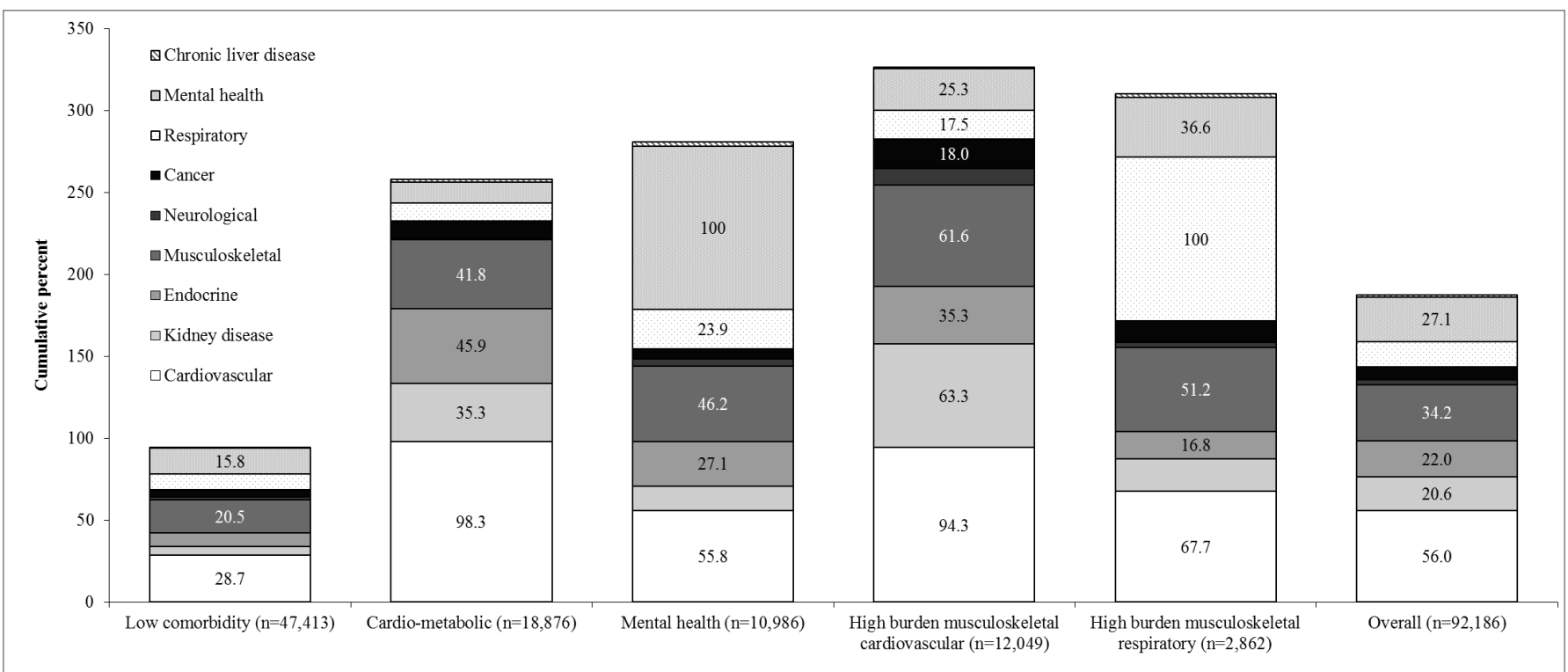

Figure 1 Cumulative percentage of groups of comorbidities according to comorbidity phenotype at the time of diagnosis of ischaemic heart disease. 


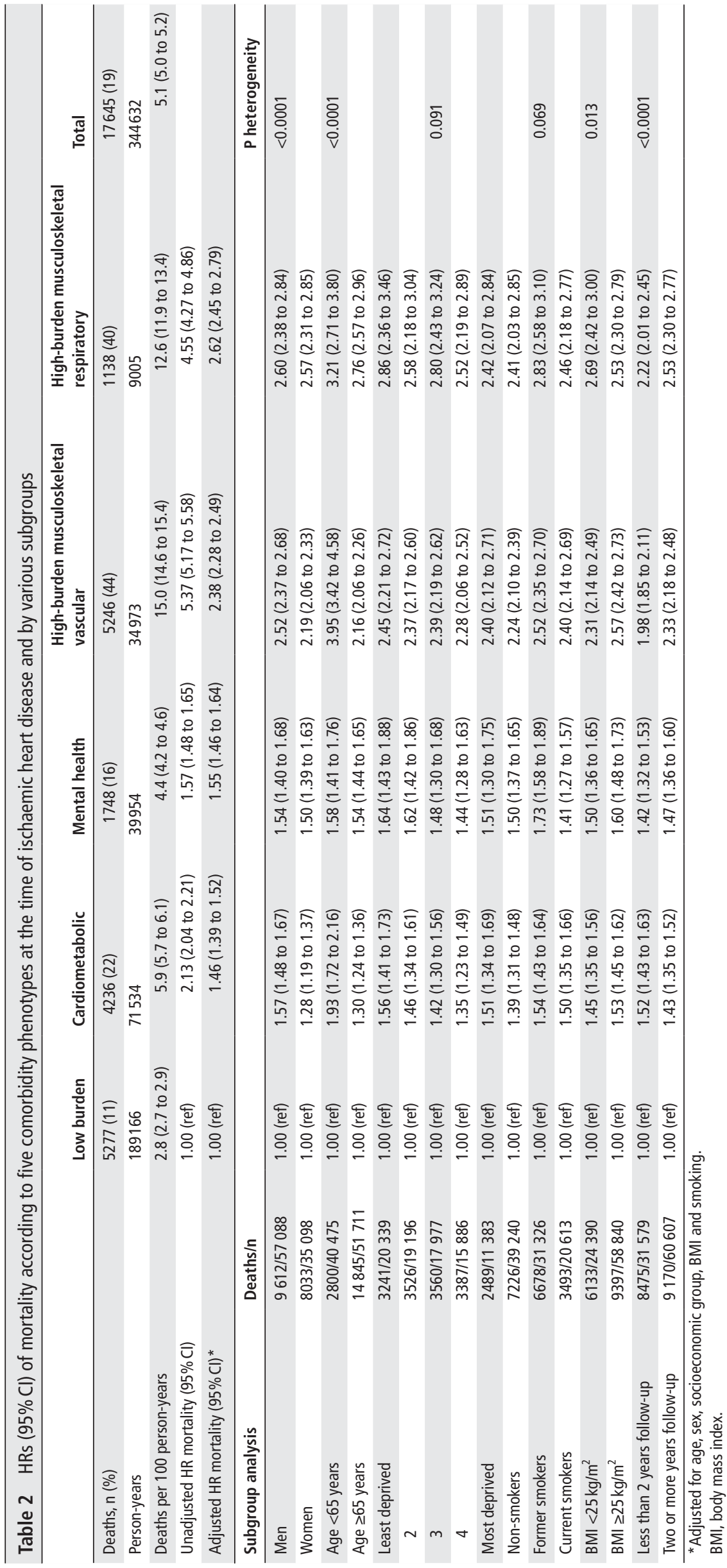




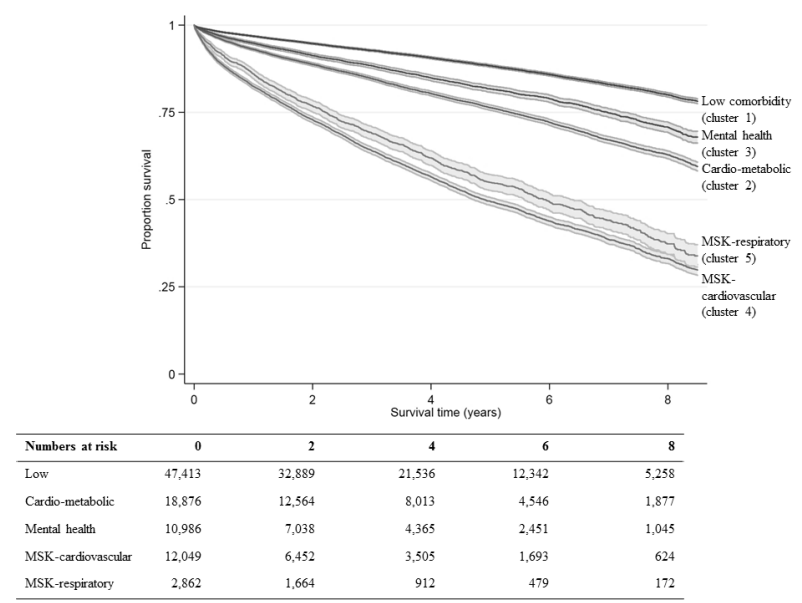

Figure 2 Long-term survival according to comorbidity phenotypes. Unadjusted Kaplan-Meier curves with $95 \% \mathrm{Cl}$ according to comorbidity phenotype (MSK: musculoskeletal).

both cardiometabolic (cluster 2) and mental health (cluster 3) was about $50 \%$ greater than the low comorbidity cluster: HR 1.46 (95\% CI 1.39 to 1.52 ) and 1.55 (95\% CI 1.46 to 1.64 ), respectively. Patients with IHD in the high burden musculoskeletal cardiovascular (cluster 4) and musculoskeletal respiratory (cluster 5) clusters had more than twice the hazard of dying compared with the low comorbidity cluster: HR 2.38 (95\% CI 2.28 to 2.49 ) and 2.62 (95\% CI 2.45 to 2.79 ), respectively.

In subgroup analysis, the hazard of mortality for each of the four comorbidity clusters was higher among younger $(<65$ years) than older patients ( $\geq 65$ years). Younger patients in the musculoskeletal cardiovascular (cluster 4) and the musculoskeletal respiratory cluster (cluster 5) had three to four times (HR 3.95, 95\% CI 3.42 to 4.58 and 3.21 , 95\% CI 2.71 to 3.80 , respectively) the hazard of dying of the low comorbidity cluster. The HR for the four comorbidity clusters was generally higher among men than women ( $p$ heterogeneity $<0.0001$ ), those with a higher versus a lower BMI $(p$ heterogeneity $=0.013)$ and those with a longer follow-up time ( $\mathrm{p}$ heterogeneity $<0.0001)$. There was no strong evidence for a difference in the HR for the four comorbidity clusters by smoking status ( $p$ heterogeneity $=0.069$ ) or socioeconomic group ( $p$ heterogeneity $=0.091$ ).

In analyses where the hazard of mortality was assessed for each of the individual comorbidities (see online supplementary table 5), having a diagnosis of dementia was associated with the highest hazards of mortality (HR 2.13, 95\% CI 1.96 to 2.32), followed by heart failure (HR $1.91,95 \%$ CI 1.82 to 2.00 ) and chronic liver disease (HR 1.91, 95\% CI 1.65 to 2.20). In sensitivity analysis, inclusion of only patients with complete data on smoking, BMI and socioeconomic group made minimal difference to the associations of the disease clusters with hazard of mortality (see online supplementary table 6). Mortality was slightly higher in all disease clusters when only patients who had a MI were included in the analysis.

\section{DISCUSSION}

In this large cohort of patients with heart disease, in which more than $80 \%$ had at least one other long-term health condition and over $60 \%$ had two or more comorbidities, we identified five comorbidity clusters that were differentially associated with the risk of dying. Patients with a high-burden musculoskeletal cardiovascular (cluster 4) and a high-burden musculoskeletal respiratory (cluster 5) phenotype had the greatest risk of mortality. IHD patients with a cardiometabolic or mental health comorbidity phenotype had a moderately elevated risk of mortality compared with those in the low comorbidity cluster.

A previous large cohort study examined the risk of mortality of hospitalised MI survivors according to comorbidity phenotypes identified using latent class analysis. ${ }^{9}$ Only seven comorbidities were considered and median follow-up was 2.3 years. By contrast, we included all patients with IHD, considered 20 comorbidities and had a slightly higher median follow-up time of 3.2 years. They identified three clusters, with the greatest risk of mortality characterised by a history of heart failure, PVD, renal failure and hypertension. These were common comorbidities in the two high-burden clusters (clusters 4 and 5) in our analysis. The exact mechanisms by which the different groupings of comorbidities affect the risk of mortality are likely to be complex and multifaceted but will probably include both the direct effect of a particular condition on mortality and indirect effects arising from the use of less intensive therapies in patients with certain comorbidities ${ }^{9}$ as our results have also demonstrated.

There is a paucity of studies examining the coexistence of cardiovascular, respiratory and musculoskeletal conditions with mortality in patients with IHD; however, others have reported that patients with a high prevalence of both cardiovascular and musculoskeletal conditions have higher mortality compared with those without any major disease conditions. ${ }^{16}$ Furthermore, other studies have shown that patients with COPD have a high prevalence of other cardiovascular comorbidities ${ }^{17}$ or go on to be diagnosed with them. ${ }^{18} 19$ Musculoskeletal conditions such as osteoarthritis and IHD share some common risk factors such as a higher BMI and physical inactivity, ${ }^{20}$ and patients with CVD and comorbid arthritis have reduced capacity for exercise compared with those without comorbidity. ${ }^{21}$ Likewise, COPD and IHD share several major risk factors (eg, age, physical inactivity and smoking) and common pathophysiological pathways such as inflammation. ${ }^{22}$ The combination of cardiovascular, respiratory and musculoskeletal diseases may lead to increased mortality by contributing to the progression of frailty in these patients ${ }^{2324}$ but more research on the effects of comorbidity patterns and frailty is needed to support this.

A systematic review has shown that in terms of multimorbidity clustering, two clusters-the cardiometabolic and mental health-are identified using a number of different statistical approaches. ${ }^{8}$ In this analysis, these clusters were associated with a moderately increased mortality risk. Mental health conditions such as depression and anxiety are frequent comorbid diagnoses in patients with heart disease. ${ }^{1}$ Patients with depression have an elevated risk of IHD partly due to the greater presence of risk factors, such as smoking and obesity, as our results have shown, but there may be other mechanisms involved. ${ }^{25}$

This analysis has highlighted the importance of common risk factors such as smoking and obesity that predispose patients to a number of different diseases and further understanding of the major determinants of these comorbidity clusters will be useful. We have identified groups of patients with a certain combination of diseases who have a higher risk of dying. If others identify these clusters and find them to be associated with higher mortality rates, then there may be a need to identify and target these complex groups of patients with coordinated multidisciplinary interventions in both primary and secondary care settings. 


\section{Strengths and limitations}

Strengths of this study include a large sample size and the inclusion of 20 different comorbidities that were taken from diagnoses recorded in primary care rather than self-reported, which has the tendency to overestimate the presence of certain health conditions. ${ }^{26}$ Patients in THIN are broadly representative of the UK population and so these results are should be generalisable.

Limitations include the possible overdiagnosis of IHD due to the extensive list of Read codes used. We examined comorbidities that were diagnosed before IHD but did not take into account the order of diagnosis of each condition. Analyses that make use of network analysis ${ }^{27}$ and disease trajectories ${ }^{28}$ may provide further insights into both the prevention and management of different comorbid clusters. Furthermore, we used an approach where the clusters were identified based on the probability of belonging to a group with similar combinations of comorbidities. A potential limitation of this method was that cluster membership was decided deterministically using posterior probabilities of belonging to the different classes. Although patients were allocated to mutually exclusive groups based on the highest observed probability of membership, some patients could belong to a different cluster where the probabilities differed by only a small amount. There is a lack of consensus over the criteria used to select the optimal number of clusters; however, as suggested by others, ${ }^{8}$ we have based our decision on at least two criteria and have attempted to justify our choice where they conflicted. If a three-cluster solution was chosen over a five-cluster solution, we may have missed identifying the two clusters with the highest mortality rates. While this method helped to identify the most common clusters of comorbidities, which may be useful if developing specific clinics for these patients, ${ }^{3}$ methods that cluster patients according to an outcome such as hospital admissions (outcome-guided) may be better at identifying the combination of comorbidities that are associated with the greatest burden on healthcare services. ${ }^{29}$ It is also important to develop a consensus

\section{Key messages}

What is already known on this subject?

- A greater burden of comorbidities in patients with heart disease increases their risk of dying.

- Most studies have not distinguished between heart disease patients with varying patterns of comorbidities.

\section{What might this study add?}

- Two groups of patients with a high burden of comorbidities had the highest risk of mortality: those with vascular and musculoskeletal conditions, HR 2.38 ( $95 \% \mathrm{Cl} 2.28$ to 2.49 ) and those with respiratory and musculoskeletal conditions, HR 2.62 ( $95 \% \mathrm{Cl} 2.45$ to 2.79 ) compared with a low-burden comorbidity group.

- Hazards of mortality in two other groups of patients characterised by cardiometabolic and mental health comorbidities were moderately higher than the low-burden comorbidity group; HR $1.46(95 \% \mathrm{Cl} 1.39$ to 1.52$)$ and 1.55 (1.46 to 1.64$)$, respectively.

\section{How might this impact on clinical practice?}

- This research may help shape the development of future interventions or health services that take into account the impact of these comorbidities in patients with ischaemic heart disease. in the methods used to derive the patterns of comorbidities so that the effect of comorbidity phenotypes can be compared between studies. ${ }^{7}$ Future studies might also test whether highburden comorbidity clusters are better predictors of mortality or emergency admissions than high-burden comorbidity counts. ${ }^{30}$

\section{CONCLUSION}

This analysis has identified five distinct comorbidity clusters in patients with IHD that were associated with varying risk of mortality. These analyses should be replicated in other large datasets, and this may help shape the development of future interventions or health services that take into account the impact of these comorbidity clusters.

\section{Twitter Dawit T Zemedikun @Dawit_TZ and Tom Marshall @TomPMarshall}

Contributors FC, TM and KN had the original idea for the study. FC, TM and KN designed the study. FC and DTZ designed and performed the analysis. $\mathrm{FC}$ wrote the first draft of the paper, which was revised in collaboration with NJA, TM, KN, KO, GR, MS and DTZ.

Funding Funding support was provided by a contract from the British Heart Foundation https://www.bhf.org.uk.

Disclaimer This paper presents independent research and the views expressed are those of the author(s) and not necessarily those of the British Heart Foundation (BHF).

\section{Competing interests None declared.}

Patient and public involvement Patients and/or the public were not involved in the design, or conduct, or reporting, or dissemination plans of this research.

\section{Patient consent for publication Not required.}

Ethics approval Collection of data for THIN was approved by the South-East Multicentre Research Ethics Committee in 2003; under the terms of this approval, studies must undergo independent scientific review. Scientific Review Committee approval for this analysis was obtained in June 2018 (SRC reference 18THIN066).

Provenance and peer review Not commissioned; externally peer reviewed.

Data availability statement All data relevant to the study are included in the article or uploaded as supplementary information. All relevant data are within the paper and its supporting information files.

Open access This is an open access article distributed in accordance with the Creative Commons Attribution 4.0 Unported (CC BY 4.0) license, which permits others to copy, redistribute, remix, transform and build upon this work for any purpose, provided the original work is properly cited, a link to the licence is given, and indication of whether changes were made. See: https://creativecommons.org/ licenses/by/4.0/.

\section{ORCID iDs}

Francesca Crowe http://orcid.org/0000-0003-4026-1726

Dawit T Zemedikun http://orcid.org/0000-0003-3642-0456

Nicola Jaime Adderley http://orcid.org/0000-0003-0543-3254

\section{REFERENCES}

1 Tran J, Norton R, Conrad N, et al. Patterns and temporal trends of comorbidity among adult patients with incident cardiovascular disease in the UK between 2000 and 2014: a population-based cohort study. PLoS Med 2018;15:e1002513.

2 Kontis V, Bennett JE, Mathers CD, et al. Future life expectancy in 35 industrialised countries: projections with a Bayesian model ensemble. Lancet 2017;389:1323-35.

3 Wallace E, Salisbury C, Guthrie B, et al. Managing patients with multimorbidity in primary care. BMJ 2015;350:h176.

4 Rashid M, Kwok CS, Gale CP, et al. Impact of co-morbid burden on mortality in patients with coronary heart disease, heart failure, and cerebrovascular accident: a systematic review and meta-analysis. Eur Heart J Qual Care Clin Outcomes 2017:3:20-36

5 , Di Angelantonio E, Kaptoge $\mathrm{S}$, et al, Emerging Risk Factors Collaboration. Association of cardiometabolic multimorbidity with mortality. JAMA 2015;314:52-60.

6 Raina P, Gilsing A, Freisling $\mathrm{H}$, et al. The combined effect of cancer and cardiometabolic conditions on the mortality burden in older adults. J Gerontol A Biol Sci Med Sci 2019:74:366-72.

7 Ng SK, Tawiah R, Sawyer M, et al. Patterns of multimorbid health conditions: a systematic review of analytical methods and comparison analysis. Int J Epidemiol 2018;47:1687-704. 
8 Busija L, Lim K, Szoeke C, et al. Do replicable profiles of multimorbidity exist? systematic review and synthesis. Eur J Epidemiol 2019;34:1025-53. Article in press.

9 Hall M, Dondo TB, Yan AT, et al. Multimorbidity and survival for patients with acute myocardial infarction in England and Wales: latent class analysis of a nationwide population-based cohort. PLoS Med 2018;15:e1002501.

10 Blak BT, Thompson M, Dattani H, et al. Generalisability of the health improvement network (thin) database: demographics, chronic disease prevalence and mortality rates. Inform Prim Care 2011;19:251-5.

11 Booth N. What are the read codes? Health Libr Rev 1994;11:177-82.

12 Maguire A, Blak BT, Thompson M. The importance of defining periods of complete mortality reporting for research using automated data from primary care. Pharmacoepidemiol Drug Saf 2009;18:76-83.

13 Horsfall L, Walters K, Petersen I. Identifying periods of acceptable computer usage in primary care research databases. Pharmacoepidemiol Drug Saf 2013;22:64-9.

14 Springate DA, Kontopantelis E, Ashcroft DM, et al. ClinicalCodes: an online clinical codes repository to improve the validity and reproducibility of research using electronic medical records. PLoS One 2014;9:e99825.

15 Levey AS, Stevens LA, Schmid CH, et al. A new equation to estimate glomerular filtration rate. Ann Intern Med 2009;150:604-12.

16 Willadsen TG, Siersma V, Nicolaisdóttir DR, et al. Multimorbidity and mortality: a 15-year longitudinal registry-based nationwide Danish population study. J Comorb 2018;8:2235042X18804063.

17 Chen W, Thomas J, Sadatsafavi M, et al. Risk of cardiovascular comorbidity in patients with chronic obstructive pulmonary disease: a systematic review and meta-analysis. Lancet Respir Med 2015;3:631-9.

18 Tényi Ákos, Vela E, Cano I, et al. Risk and temporal order of disease diagnosis of comorbidities in patients with COPD: a population health perspective. BMJ Open Respir Res 2018:5:e000302.

19 Dursunoğlu N, Köktürk N, Baha A, et al. Comorbidities and their impact on chronic obstructive pulmonary disease. Tuberk Toraks 2016;64:289-98.
20 Fernandes GS, Valdes AM. Cardiovascular disease and osteoarthritis: common pathways and patient outcomes. Eur J Clin Invest 2015;45:405-14.

21 Slater M, Perruccio AV, Badley EM. Musculoskeletal comorbidities in cardiovascular disease, diabetes and respiratory disease: the impact on activity limitations; a representative population-based study. BMC Public Health 2011;11:77.

22 Bhatt SP, Dransfield MT. Chronic obstructive pulmonary disease and cardiovascular disease. Trans/ Res 2013;162:237-51.

23 Pollack LR, Litwack-Harrison S, Cawthon PM, et al. Patterns and predictors of frailty transitions in older men: the osteoporotic fractures in men study. J Am Geriatr Soc 2017;65:2473-9.

24 Kojima G, lliffe S, Walters K. Frailty index as a predictor of mortality: a systematic review and meta-analysis. Age Ageing 2018;47:193-200.

25 Vaccarino V, Badimon L, Bremner JD, et al. Depression and coronary heart disease: 2018 ESC position paper of the working group of coronary pathophysiology and microcirculation developed under the auspices of the ESC committee for practice guidelines. Eur Heart J 2019:ehy913.

26 Gontijo Guerra S, Berbiche D, Vasiliadis H-M. Measuring multimorbidity in older adults: comparing different data sources. BMC Geriatr 2019;19:166.

27 Hidalgo CA, Blumm N, Barabási A-L, et al. A dynamic network approach for the study of human phenotypes. PLoS Comput Biol 2009;5:e1000353.

28 Jensen AB, Moseley PL, Oprea TI, et al. Temporal disease trajectories condensed from population-wide registry data covering 6.2 million patients. Nat Commun 2014;5:4022.

29 Liverani S, Hastie DI, Azizi L, et al. PReMiuM : An R Package for Profile Regression Mixture Models Using Dirichlet Processes. J Stat Softw 2015;64:1-30.

30 Wallace E, McDowell R, Bennett K, et al. Comparison of count-based multimorbidity measures in predicting emergency admission and functional decline in older community-dwelling adults: a prospective cohort study. BMJ Open 2016;6:e013089. 